01

\title{
Изменение теплофизических свойств алмаза при изотермическом сжатии
}

\author{
(C) М.Н. Магомедов
}

Институт проблем геотермии Дагестанского научного центра РАН, 367030 Махачкала, Россия

e-mail: mahmag4@mail.ru

(Поступило в Редакцию 23 июня 2015 г.)

Исходя из парного межатомного потенциала Ми-Леннард-Джонса и модели кристалла Эйнштейна, без каких-либо подгоночных параметров получены уравнение состояния $P\left(V / V_{0}, T\right)$ и барические зависимости теплофизических свойств алмаза. Расчеты выполнены вдоль двух изотерм: $T=300$ и $3000 \mathrm{~K}$ до $P=10000 \mathrm{kbar}=1000 \mathrm{GPa}$ (т. е. до относительного объема $V / V_{0}=0.5$ ). Получены барические зависимости следующих свойств: $B_{T}-$ изотермического модуля упругости и $B^{\prime}(P), C_{v}-$ изохорной теплоемкости и $C_{v}^{\prime}(P), C_{p}$ - изобарной теплоемкости, $\alpha_{p}-$ коэффициента теплового расширения и $\alpha_{p}^{\prime}(P), \sigma-$ удельной поверхностной энергии, а также производных $\sigma^{\prime}(P)$ и $\sigma^{\prime}(T)$. Показано, что при $P \rightarrow \infty$ функции $B_{T}(P)$ и $\sigma(P)$ изменяются по линейной зависимости, функции $B^{\prime}(P), \alpha_{p}(P), C_{v}(P), C_{p}(P)$ и $\sigma^{\prime}(P)$ стремятся к постоянным величинам, а функции $\alpha_{p}^{\prime}(P), C_{v}^{\prime}(P)$ и разность $C_{p}(P)-C_{v}(P)$ стремятся к нулю. Получено хорошее согласие с экспериментальными данными.

DOI: 10.21883/JTF.2017.05.44434.1485

\section{Введение}

Зависимость свойств вещества от давления изучается уже давно, но до сих пор осталась неясной зависимость коэффициента теплового объемного расширения: $\alpha_{p}=(\partial \ln V / \partial T)_{P}$ и теплоемкости от величины гидростатического давления $(P)$ вдоль различных изотерм при высоких давлениях (при $P>1000 \mathrm{kbar}=100 \mathrm{GPa}$ ) [1,2]. Используемые для изучения уравнения состояния кристалла при высоких давлениях различные (как численные, так и аналитические) методы содержат в своем формализме подгоночные константы, которые лишены физического смысла, и поэтому предсказать их барическую зависимость вдоль изотермы очень затруднительно. Это делает результаты, полученные такими методами, неудобными для дальнейших приложений.

Между тем множество прикладных задач нуждаются в ясных аналитических зависимостях термодинамических свойств от давления. Поэтому в настоящей работе будет показано, что как уравнение состояния, так и барические зависимости решеточных свойств кристалла простого однокомпонентного вещества можно с достаточной точностью рассчитать сравнительно простым аналитическим методом без использования различных компьютерных моделей и без введения лишенных физического смысла подгоночных констант.

Алмаз является одним из основных материалов, используемых в технике высоких давлений. Именно алмазные наковальни являются основным инструментом для изучения вещества при высоких $P-T$-условиях. Между тем барические зависимости теплофизических свойств алмаза исследованы сравнительно мало. В связи с этим в настоящей работе будет получено как уравнение состояния, так и изменение теплофизических свойств при всестороннем сжатии алмаза вдоль изотерм как при низких, так и при высоких температурах. Несмотря на простоту методики расчета, получено хорошее согласие с экспериментальными данными.

\section{Метод расчета термодинамических свойств}

Представим парное межатомное взаимодействие в кристалле однокомпонентного вещества в виде потенциала Ми-Леннард-Джонса, имеющего вид

$$
\varphi(r)=\frac{D}{(b-a)}\left[a\left(\frac{r_{0}}{r}\right)^{b}-b\left(\frac{r_{0}}{r}\right)^{a}\right],
$$

где $D$ и $r_{0}$ - глубина и координата минимума потенциала, $b>a \geq 1$ - параметры.

Тогда температуру Дебая можно определить из выражения $[3,4]$

$$
\Theta=A_{w} \xi\left[-1+\left(1+\frac{8 D}{k_{B} A_{w} \xi^{2}}\right)^{1 / 2}\right],
$$

где $k_{B}$ - постоянная Больцмана, функция $A_{w}$ возникает из-за учета энергии „нулевых колебаний“ атомов в кристалле:

$$
\begin{gathered}
A_{w}=K_{R} \frac{5 k_{n} a b(b+1)}{144(b-a)}\left(\frac{r_{0}}{c}\right)^{b+2}, \\
K_{R}=\frac{\hbar^{2}}{k_{B} r_{0}^{2} m}, \quad \xi=\frac{9}{k_{n}},
\end{gathered}
$$

$m$ - масса атома, $\hbar$ - постоянная Планка, $k_{n}$ первое координационное число, $c=\left[6 k_{p} V /(\pi N)\right]^{1 / 3}-$ 
расстояние между центрами ближайших атомов, $k_{p}-$ коэффициент упаковки структуры, $V$ и $N-$ объем и число атомов в кристалле простого вещества.

Используя для колебательного спектра одноатомного кристалла модель Эйнштейна и приближение „взаимодействия только ближайших соседей“, для удельной свободной энергии Гельмгольца можно принять выражение [5]

$$
\begin{aligned}
\frac{F}{N}= & \left(\frac{k_{n}}{2}\right) D U(R)+3 k_{B} \Theta_{E}\left\{\left(\frac{1}{2}\right)\right. \\
& \left.+\left(\frac{T}{\Theta_{E}}\right) \ln \left[1-\exp \left(-\frac{\Theta_{E}}{T}\right)\right]\right\},
\end{aligned}
$$

где $\Theta_{E}-$ это температура Эйнштейна, которая связана с температурой Дебая соотношением [5]: $\Theta=(4 / 3) \Theta_{E}$, $R=r_{0} / c$ - линейная относительная плотность кристалла, функция потенциальной энергии в соответствии с (1) равна

$$
U(R)=\frac{a R^{b}-b R^{a}}{b-a} .
$$

Исходя из (2)-(4) можно рассчитать решеточные свойства кристалла при данных значениях $V / N$ и $T$, если известны параметры межатомного потенциала (1) и структура кристалла. Для термического уравнения состояния $(P)$ и изотермического модуля упругости $\left(B_{T}\right)$ можно получить

$$
\begin{aligned}
P=-\left(\frac{\partial F}{\partial V}\right)_{T} & =\left[\frac{k_{n}}{6} D U^{\prime}(R)\right. \\
& \left.+3 k_{B} \Theta_{E}(R) \gamma(R) E_{w}(y)\right] \frac{N}{V}, \\
B_{T}= & -V\left(\frac{\partial P}{\partial V}\right)_{T}=P+\left[\frac{k_{n}}{18} D U^{\prime \prime}(R)\right. \\
& \left.+3 k_{B} \Theta_{E} \gamma(\gamma-q) E_{w}(y)-\gamma^{2} T \frac{C_{v}(y)}{N}\right] \frac{N}{V} .
\end{aligned}
$$

Здесь $C_{v}$ - изохорная теплоемкость кристалла по модели Эйнштейна, которая определяется выражением [5]

$$
\begin{gathered}
C_{v}=3 N k_{B} \frac{y^{2} \exp (y)}{[\exp (y)-1]^{2}}, \\
E_{w}(y)=0.5+\frac{1}{[\exp (y)-1]}, \quad y=\frac{\Theta_{E}}{T}=\frac{3 \Theta}{4 T}, \\
U^{\prime}(R)=R\left[\frac{\partial U(R)}{\partial R}\right]=\frac{a b\left(R^{b}-R^{a}\right)}{b-a}, \\
U^{\prime \prime}(R)=R\left[\frac{\partial U^{\prime}(R)}{\partial R}\right]=\frac{a b\left(b R^{b}-a R^{a}\right)}{b-a} .
\end{gathered}
$$

В соответствии с (2) первый, второй и третий параметры Грюнайзена имеют вид

$$
\gamma=-\left(\frac{\partial \ln \Theta}{\partial \ln V}\right)_{T}=\frac{b+2}{6\left(1+X_{w}\right)}, \quad X_{w}=\frac{A_{w} \xi}{\Theta},
$$

$$
\begin{gathered}
q=\left(\frac{\partial \ln \gamma}{\partial \ln V}\right)_{T}=\gamma \frac{X_{w}\left(1+2 X_{w}\right)}{\left(1+X_{w}\right)} \\
z=-\left(\frac{\partial \ln q}{\partial \ln V}\right)_{T}=\gamma\left(1+4 X_{w}\right)-2 q \\
=\gamma\left(\frac{1+3 X_{w}}{1+X_{w}}\right)=\frac{(b+2)}{6} \frac{\left(1+3 X_{w}\right)}{\left(1+X_{w}\right)^{2}} .
\end{gathered}
$$

Изобарный коэффициент теплового объемного расширения можно рассчитать по уравнению Грюнайзена [5]

$$
\alpha_{p}=\frac{\gamma C_{v}}{V B_{T}}=\frac{\gamma C_{v}}{N B_{T}\left[\pi r_{0}^{3} /\left(6 k_{p}\right)\right]}\left(\frac{V_{0}}{V}\right), V_{0}=N \frac{\pi r_{0}^{3}}{6 k_{p}} .
$$

Изобарную теплоемкость определим в виде [5] $C_{p}=C_{v}\left(1+\gamma \alpha_{p} T\right)$.

Удельную (на единицу площади) поверхностную энергию грани (100) будем рассчитывать по формуле $[3,6]$

$$
\sigma=-\frac{k_{n} D R^{2}}{12 \alpha^{2 / 3} r_{0}^{2}}\left[U(R)+3 H_{w}(R, T)\right],
$$

где введены обозначения $\alpha=\pi /\left(6 k_{p}\right)$,

$$
H_{w}(R, T)=\frac{6 \gamma(R)}{(b+2)}\left[\frac{k_{B} \Theta_{E}(R)}{D k_{n}}\right] E_{w}\left(\frac{\Theta_{E}}{T}\right) .
$$

Таким образом, полученные выражения (2)-(10) позволяют рассчитать зависимость как уравнения состояния, так и указанных теплофизических свойств от аргументов $V / V_{0}=\left(c / r_{0}\right)^{3}=R^{-3}$ и $T$ для данной структуры одноатомного кристалла (т.е. при данных $k_{n}$ и $\left.k_{p}\right)$, если известны все четыре параметра межатомного потенциала (1).

Из зависимостей (2), (3) и (8) видно, что при $V / V_{0} \rightarrow 0$ (т.е. при $X_{w} \rightarrow \infty$ или $P \rightarrow \infty$ ) выполняются следующие предельные соотношения:

$$
\begin{aligned}
& \lim _{V / V_{0} \rightarrow 0} \Theta=\Theta_{\max }=\frac{4 k_{n} D}{9 k_{B}}, \quad \lim _{V / V_{0} \rightarrow 0} \gamma=\gamma_{\min }=0, \\
& \lim _{V / V_{0} \rightarrow 0} q=q_{\max }=\frac{b+2}{3}, \quad \lim _{V / V_{0} \rightarrow 0} z=z_{\min }=0 .
\end{aligned}
$$

При этом с ростом давления функция $z\left(X_{w}\right)$ сначала достигает максимума при $X_{w}=1 / 3: z_{\max }=3(b+2) / 16$, после чего она уменьшается до нуля $z_{\min }=0$.

\section{О параметрах межатомного потенциала для алмаза}

Для расчетов возьмем алмаз $m(\mathrm{C}$-dia $)=12.01$ a.m.u., $k_{n}=4, \quad k_{p}=0.3401, \quad \xi=2.25, \alpha=1.5396$. Значение $r_{0}$-координаты минимума потенциала (1) для алмаза определено в [3,7] из мольного объема при $T=0 \mathrm{~K}$ и $P=0: V_{0}($ C-dia $)=3.42 \mathrm{~cm}^{3} / \mathrm{mol}[8]$.

Так как для алмаза при $R=r_{0} / c=\left(V_{0} / V\right)^{1 / 3}=1$ выполняется: $X_{w}(1)=A_{w}(1) \xi / \Theta(1) \ll 1$, то $b-$ параметр жесткости межатомного потенциала (1) с хорошей 
Таблица 1. Параметры межатомного потенциала Ми-Леннард-Джонса (1) для алмаза и рассчитанные по ним при $R=1$ температура Дебая, параметры Грюнайзена и величина $X_{w}(1)$. Также представлены значения для максимума температуры Дебая при $V / V_{0}=0$ и величина $T_{\sigma}$ — „температура фрагментации“ кристалла. В первой строке представлены результаты для $D_{b}$, а во второй строке - для $D_{s}$

\begin{tabular}{c|c|c|c|c|c|c|c|c|c|c}
\hline$r_{0}, 10^{-10} \mathrm{~m}$ & $b$ & $a$ & $D / k_{B}, \mathrm{~K}$ & $\Theta(1), \mathrm{K}$ & $\gamma(1)$ & $q(1) \cdot 10^{3}$ & $z(1)$ & $X_{w}(1) \cdot 10^{3}$ & $\Theta_{\max }, \mathrm{K}$ & $T_{\sigma}, \mathrm{K}$ \\
\hline 1.545 & 3.79 & 2.05 & 97821.72 & 1972.04 & 0.9595 & 5.534 & 0.970 & 5.735 & 173905 & 130429 \\
& & & 42702.72 & 1299.14 & 0.9567 & 8.400 & 0.973 & 8.705 & 75916 & 56937
\end{tabular}

точностью был определен в [3,7] с помощью (8) из первого параметра Грюнайзена при $T=0 \mathrm{~K}$ и $P=0$ : $\gamma_{0}(\mathrm{C}$-dia $)=0.965[8]$. Эти значения $r_{0}$ и $b$ представлены в табл. 1. Исходя из данного $b$ значения максимумов для второго и третьего (при $X_{w}=1 / 3$ ) параметров Грюнайзена алмаза будут равны: $q_{\max }\left(V / V_{0}=0\right)=1.93$ и $z_{\max }\left(X_{w}=1 / 3\right)=1.085625$.

Что касается $D$ - глубины потенциала (1), то, как было показано в $[3,7,9]$, эта величина для ковалентных кристаллов (в отличие от металлов) существенно зависит от вида деформации, которой подвергается кристалл (упругой или пластической). Это обусловлено тем, что ковалентная связь состоит из двух звеньев: сильного и слабого, причем энергия слабого звена приблизительно вдвое меньше, чем сильного. Энергия всей межатомной связи $\left(D_{b}\right.$, которая работает при упругой деформации) и энергия слабой связи $\left(D_{s}\right.$, которая рвется при пластической деформации) равны

$$
D_{b}=\frac{18 B_{0} V_{0}}{k_{n} a b N}=D_{s}+\Delta D, \quad D_{s}=\frac{L_{0}}{k_{n} / 2},
$$

где $L_{0}$ и $B_{0}-$ удельная энергия сублимации и модуль упругости при $T=0 \mathrm{~K}$ и $P=0: L_{0}(\mathrm{C}$-dia $)=7.36 \mathrm{eV}$, $B_{0}(\mathrm{C}$-dia $)=4430 \mathrm{kbar}[3,7]$.

В (12) величина $D_{s} / 2=L_{0} / k_{n}$ - это энергия связи электрона с „чужим“ ионом, т.е. это одно из двух звеньев слабой связи. Величина $\Delta D / 2=\left(D_{b}-\right.$ $\left.-D_{s}\right) / 2=D_{s} / 2+d-$ это энергия связи электрона со „своим“ ионом или одно из двух звеньев сильной связи. Разница между энергиями сильной и слабой связей $(d)$ определяет энергию, благодаря которой обобщенный в ковалентной связи валентный электрон локализуется вблизи „своего“ иона.

Как было показано в $[3,7,9]$, при упругой (обратимой) деформации ковалентных кристаллов работают одновременно сильное и слабое звенья ковалентной связи и глубина межатомного потенциала равна $D_{b}$. Именно из значения $D_{b}$ и необходимо рассчитывать такие параметры, при измерении которых не происходит разрыва межатомных связей: скорость звука, температура Дебая, коэффициент теплового расширения. При пластической (необратимой) деформации ковалентного кристалла рвутся только слабые звенья связи, и глубина потенциала определяется величиной $D_{s}$. Поэтому из величины $D_{s}$ определяются такие (связанные с разрывом межатомных связей) параметры, как энергия сублимации $L_{0}$ и энергия активационных процессов (энергия образования вакансий и самодиффузии), удельная поверхностная энергия. В табл. 1 представлены два значения глубины потенциала (1): в первой строке $D_{b}-$ для упругой (обратимой) деформации, а во второй строке $D_{s}$ - для пластической (необратимой) деформации алмаза.

Первоначально в [3,7] величина $a$ - степень притяжения потенциала (1) рассчитывалась из величины $B^{\prime}(P=0)=\left(\partial B_{T} / \partial P\right)_{T=0 \mathrm{~K}}-$ производной модуля упругости по давлению вдоль изотермы $T=0 \mathrm{~K}$ при $P=0$, т.е. из формулы $a=3\left(B^{\prime}(P=0)-2\right)-b$. Отсюда для алмаза при $B^{\prime}(P=0)=4$ [8] было получено $a=2.21[3,7]$. Однако, как было указано в [8], величина $B^{\prime}(P=0)$ для алмаза измеряется с большой погрешностью. Поэтому в настоящей работе величина $a$ была скорректирована по величине $\alpha_{p}(P, T)$, измеренной при нормальных условиях, т.е. при $P=0$ и $T=300 \mathrm{~K}$. Для алмаза величина $\alpha_{p}(0,300 \mathrm{~K})$ измерена с высокой (по сравнению с $B^{\prime}(P=0)$ ) точностью и равна

$\alpha_{p}(0,300 \mathrm{~K}) /\left[10^{-6} \mathrm{~K}^{-1}\right]=3.0[10], 3.15$ [11], 3.0507 [12].

Именно подгонка под это значение и дала величину $a=2.05$, которая представлена в табл. 1, и которая была использована нами при расчете как функции $P\left(V / V_{0}\right)$, так и барических зависимостей теплофизических свойств алмаза. Заметим, что полученное значение $a=2.05$ лишь на $8 \%$ меньше величины $a=2.21$ из $[3,7]$, которая была использована в [9] для расчета температурной зависимости функций $\alpha_{p}, B_{T}, C_{v}$ и $C_{p}$ при $P=0$. Можно полагать, что именно поэтому в $[9]$ было получено хорошее согласие с экспериментальными данными.

В табл. 1 представлены как параметры межатомного потенциала (1) для алмаза, так и рассчитанные из них при $R=1$ значения: температуры Дебая, первого, второго и третьего параметров Грюнайзена, а также величины $X_{w}(1)=A_{w}(1) \xi / \Theta(1)$. Там же представлены значения $\Theta_{\max }$ - максимума температуры Дебая при $V / V_{0}=0$ из (11) и величины $T_{\sigma}=k_{n} D /\left(3 k_{B}\right)$ - „температура фрагментации“, выше которой алмаз фрагментируется при любом давлении [13]. В первой строке представлены результаты для упругой (обратимой) деформации (т.е. полученные из $\left.D_{b}\right)$, а во второй строке - для пла- 

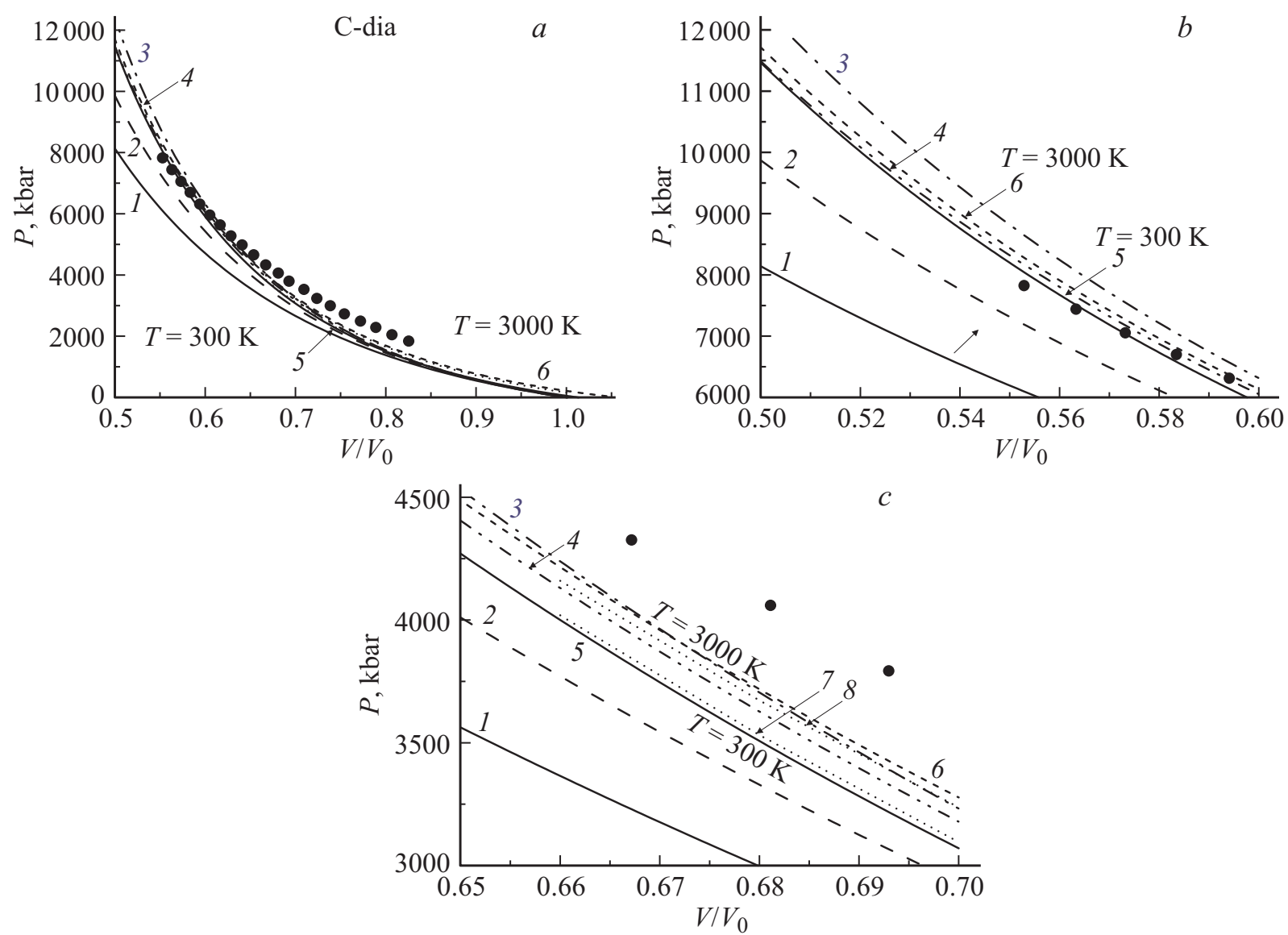

Рис. 1. Изотермы уравнения состояния алмаза. Точки - экспериментальные данные из [14, рис. 4]. Линии 1 и 2 уравнение Вине (13): сплошная линия 1 из [16], а штриховая линия 2 - из [14]. Штрихпунктирная линия 3 - уравнение Мурнагана-Берча (14) с параметрами из [3,7]. Двухштрихпунктирная линия 4 - уравнение Хольцапфеля (15) с параметрами из [17]. Линии 5 и $6-$ изотермы, рассчитанные нами с потенциалом упругого типа: $5-300 \mathrm{~K}, 6-3000 \mathrm{~K}$. Линии 7 и 8 на графике $c$ - изотермы из [2]: $7-298.15 \mathrm{~K}, 8-3000 \mathrm{~K}$.

стической (необратимой) деформации (т.е. полученные из $\left.D_{s}\right)$.

Для алмаза экспериментальные оценки температуры Дебая и параметра Грюнайзена лежат в интервале $\Theta=1860-2239 \mathrm{~K}[3]$ и $\gamma=0.965 \pm 0.005$ [8]. Из сравнения видно, что с экспериментальными данными лучше согласуются результаты, полученные при использовании величины $D_{b}$, т.е. с межатомным потенциалом для упругой деформации. Поэтому подгонка величины $a$, а потом и расчеты теплофизических свойств алмаза осуществлялись исходя из значения $D_{b}$.

Отметим, что в выражениях (2)-(10) не учитываются ни вакансии, ни самодиффузия атомов, ибо, как показано в [13], их влияние при сжатии алмаза становится пренебрежимо малым. Здесь, так же как и в $[9,13]$, не учитывается вклад в термодинамические параметры электронной подсистемы, ибо потенциал (1) описывает парное взаимодействие нейтральных атомов. Возникает вопрос: насколько точны будут расчеты по представленным здесь сравнительно простым аналитическим выражениям? Ответ на этот вопрос применительно к алмазу содержится ниже.

\section{Результаты расчетов для алмаза}

Как было экспериментально показано в [14], алмаз вплоть до $8000 \mathrm{kbar}=800 \mathrm{GPa}$ сохраняет свою кристаллическую модификацию. Поэтому расчеты для алмаза были проведены нами вдоль двух изотерм $T=300$ и $3000 \mathrm{~K}$, при сжатии до $V / V_{0}=0.5$.

На рис. 1 показаны изотермы уравнения состояния алмаза, полученные различными методами. График $a$ показывает общий вид зависимостей $P\left(V / V_{0}, T\right)$, а графики $b$ и $c$ показывают два участка графика $a$ в более увеличенном виде. Точки - это экспериментальные данные из [14, рис. 4], полученные ударным сжатием алмаза до $8000 \mathrm{kbar}$ в квазиадиабатическом режиме (quasiisentropic compression).

Две нижние линии 1 и 2 - аппроксимация экспериментальных данных уравнением Вине (P. Vinet) [15], которое имеет вид

$$
P(x)=3 B_{0}\left[(1-x) / x^{2}\right] \exp \left[1.5\left(B_{0}^{\prime}-1\right)(1-x)\right],
$$

где $x=\left(V / V_{0}\right)^{1 / 3}=1 / R$. Нижняя сплошная линия $I$ получена в $[16]$ при $B_{0}=4460 \mathrm{kbar}$ и $B_{0}^{\prime}=3.0$. Данные 
значения получены в [16] из экспериментальных данных по сжатию алмаза гидростатическим давлением вплоть до 1400 kbar. Штриховая линия 2 получена из (13) в работе [14] при $B_{0}=4380 \mathrm{kbar}$ и $B_{0}^{\prime}=3.68$.

Верхняя штрихпунктирная линия 3 - расчет по уравнению Мурнагана-Берча (F. Birch-F.D. Murnaghan) следующего вида [17]:

$$
\begin{aligned}
P(x)= & 1.5 B_{0}\left[\left(1-x^{2}\right) / x^{7}\right] \\
& \times\left[1+(3 / 4)\left(B_{0}^{\prime}-4\right)\left(1-x^{2}\right) / x^{2}\right]
\end{aligned}
$$

при $B_{0}=4330 \mathrm{kbar}$ и $B_{0}^{\prime}=4.0[3,7]$.

Двухштрихпунктирная линия 4 - расчет по уравнению Хольцапфеля (W.B. Holzapfel) $[1,17]$

$$
P(x)=3 B_{0}\left[(1-x) / x^{5}\right] \exp \left[1.5\left(B_{0}^{\prime}-3\right)(1-x)\right]
$$

при $B_{0}=4560 \mathrm{kbar}$ и $B_{0}^{\prime}=3.8$ [17].

Линии 5 и 6 в центре графиков $b$ и $c$ - это изотермы, рассчитаны нами с потенциалом упругого типа (т.е. с $D_{b}$ ): сплошная линия 5 - изотерма $300 \mathrm{~K}$, пунктирная линия 6 - изотерма $3000 \mathrm{~K}$. Пунктирные линии 7 и 8 на графике $c$ - изотермы из работы [2], рассчитанные на интервале $V / V_{0}=0.66-1$ : нижняя линия 7 изотерма $298.15 \mathrm{~K}$, верхняя линия 8 - изотерма $3000 \mathrm{~K}$.

Из рис. 1 видно, что наши результаты достаточно хорошо согласуются как с экспериментальными данными из $[14$, рис. 4], так и с оценками других авторов. Отметим также, что наша изотерма $300 \mathrm{~K}$ хорошо согласуются с изотермой, рассчитанной в работе $[18$, рис. 1$]$ методом функционала плотности в квазигармоническом приближении на интервале $P=0-10000 \mathrm{kbar}$, т. е. для $V / V_{0}=1-0.5$.

Рассчитанные нами зависимости для $\Theta$ - температуры Дебая, $\gamma$ и $q-$ первого и второго параметров Грюнайзена от аргумента $V / V_{0}$ для алмаза были представлены в [13] и также показали хорошее согласие с данными, которые известны из литературы.

Рассчитав зависимость $P\left(V / V_{0}\right)$ и зависимость какого-либо параметра $X\left(V / V_{0}\right)$ вдоль определенной изотермы, можно получить барическую зависимость этого параметра $X(P)$ вдоль этой же изотермы. На рис. 2 сверху показаны полученные таким путем для алмаза изотермы барических зависимостей для $B_{T}(P)$ - изотермического модуля упругости - верхний график и $B^{\prime}(P)=$ $=\left(\partial B_{T} / \partial P\right)_{T}-$ производной модуля упругости по давлению - нижний график. Сплошная линия 1 и штриховая линия 2 - рассчитанные нами изотермы 300 и $3000 \mathrm{~K}$. Символы — результаты из [2, табл. 1А]: квадраты - для $298.15 \mathrm{~K}$, кружки - для $3000 \mathrm{~K}$. На верхнем графике пунктирная линия 3 на интервале $P=0-1000 \mathrm{kbar}$ - результат из [18, рис. 6], полученный методом функционала плотности в квазигармоническом приближении. Около вертикальных осей сплошными квадратами с вертикальной линией показаны области разброса экспериментальных данных, полученных для алмаза при $T=300 \mathrm{~K}$ и $P=0$ :
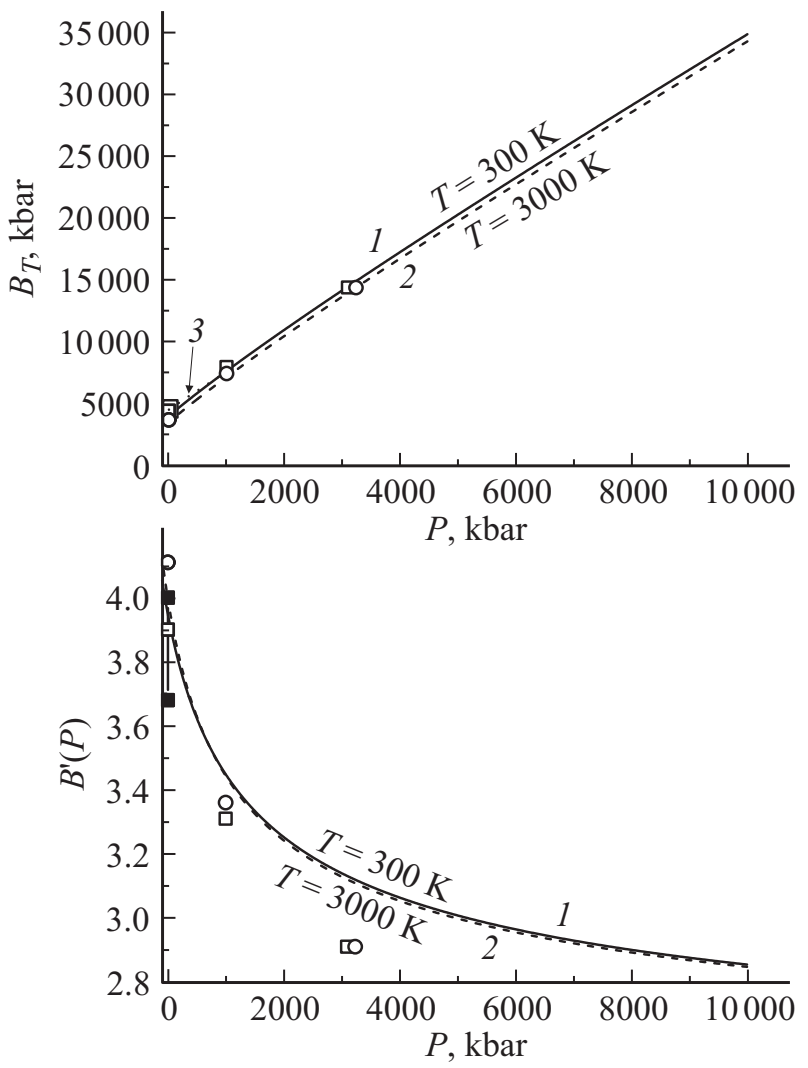

Рис. 2. Изотермы барических зависимостей для алмаза: $B_{T}(P)$ - модуль упругости - верхний график и $B^{\prime}(P)-$ производная модуля упругости по давлению (нижний график).

$B_{T}(P=0)=4380-4560 \mathrm{kbar}$ и $B^{\prime}(P=0)=3.68-4.00$. Из рис. 2 видно, что при $P \rightarrow \infty$ функция $B_{T}(P)$ выходит на линейную зависимость, а функция $B^{\prime}(P)$ стремится к постоянной величине. Из графика зависимости $B^{\prime}(P)$ видно, что в определенной точке: $P_{x}=690 \mathrm{kbar}$, $B^{\prime}\left(P_{x}\right)=3.546$, изотермы пересекаются. Это указывает на то, что при этом давлении функция $B^{\prime}(P)$ не зависит от температуры, при $P<P_{x}$ функция $B^{\prime}(P)$ возрастает при изобарическом росте температуры, а при $P>P_{x}$ функция $B^{\prime}(P)$ убывает с температурой.

На рис. 3 слева показаны изотермы барической зависимости $\alpha_{p}(P)$ - коэффициента теплового расширения (в $\left.10^{-6} / \mathrm{K}\right)$, а справа - изотермы зависимости $\alpha_{p}^{\prime}(P)=\left(\partial \alpha_{p} / \partial P\right)_{T}-$ производной $\alpha_{p}(P)$ по давлению (в $\left.10^{-9} /(\mathrm{K} \cdot \mathrm{kbar})\right)$. Сплошная линия 1 и штриховая линия 2 - наши расчеты для алмаза при 300 и $3000 \mathrm{~K}$. На левом графике также показаны результаты из работы [2, табл. 1А]: квадраты - для $298.15 \mathrm{~K}$, кружки - для $3000 \mathrm{~K}$. Точечная линия 3 на интервале $P=0-1000 \mathrm{kbar}-$ результат из $[18$, рис. 3$]$, полученный методом функционала плотности в квазигармоническом приближении. Около вертикальной оси сплошными квадратами показана область разброса экспериментальных данных, полученных для алмаза при $T=300 \mathrm{~K}$ и $P=0: \alpha_{p}(0,300 \mathrm{~K}) /\left[10^{-6} \mathrm{~K}^{-1}\right]=3.0-3.15$. Из рис. 3 
Таблица 2. Значения поверхностных свойств алмаза, рассчитанные в четырех точках зависимости $\sigma(P)$. Для каждой температуры в первой строке представлены результаты расчетов с глубиной потенциала $D_{b}$, а во второй строке - результаты расчетов с $D_{s}$ из табл. 1

\begin{tabular}{|c|c|c|c|c|c|c|c|c|}
\hline \multirow[b]{2}{*}{$T, \mathrm{~K}$} & \multicolumn{4}{|c|}{$P=0$} & \multicolumn{4}{|c|}{$V / V_{0}=1$} \\
\hline & $V / V_{0}$ & $\begin{array}{c}\sigma(100) \\
10^{-3} \mathrm{~J} / \mathrm{m}^{2}\end{array}$ & $\begin{array}{c}\sigma^{\prime}(T), \\
10^{-6} \mathrm{~J} /\left(\mathrm{m}^{2} \cdot \mathrm{K}\right)\end{array}$ & $\begin{array}{c}\sigma^{\prime}(P), \\
10^{-3} \mathrm{~J} /\left(\mathrm{m}^{2} \cdot \mathrm{kbar}\right)\end{array}$ & $P$, kbar & $\begin{array}{c}\sigma(100), \\
10^{-3} \mathrm{~J} / \mathrm{m}^{2}\end{array}$ & $\begin{array}{c}\sigma^{\prime}(T), \\
10^{-6} \mathrm{~J} /\left(\mathrm{m}^{2} \cdot \mathrm{K}\right)\end{array}$ & $\begin{array}{c}\sigma^{\prime}(P), \\
10^{-3} \mathrm{~J} /\left(\mathrm{m}^{2} \cdot \mathrm{kbar}\right)\end{array}$ \\
\hline \multirow{2}{*}{300} & 1.01280 & 13946.0 & -19.76 & 2.340 & 52.52 & 14064.9 & -19.22 & 2.209 \\
\hline & 1.02115 & 6033.5 & -48.47 & 2.390 & 36.75 & 6118.5 & -47.71 & 2.173 \\
\hline \multirow{2}{*}{3000} & 1.05653 & 13301.8 & -102.11 & 2.707 & 214.22 & 13815.8 & -105.68 & 2.122 \\
\hline & 1.14362 & 5309.1 & -97.76 & 3.428 & 211.21 & 5849.8 & -106.58 & 1.940 \\
\hline \multirow[b]{2}{*}{$T, \mathrm{~K}$} & \multicolumn{4}{|c|}{$\sigma(100)_{\max }$, где $\sigma^{\prime}(P)=(\partial \sigma / \partial P)_{T}=0$} & \multicolumn{4}{|c|}{$\sigma(100)_{f r}=0$} \\
\hline & $\left(V / V_{0}\right)_{\max }$ & $P_{\max }, \mathrm{kbar}$ & $\begin{array}{c}\sigma(100)_{\max }, \\
10^{-3} \mathrm{~J} / \mathrm{m}^{2}\end{array}$ & $\begin{array}{c}\sigma^{\prime}(T), \\
10^{-6} \mathrm{~J} /\left(\mathrm{m}^{2} \cdot \mathrm{kbar}\right)\end{array}$ & $\left(V / V_{0}\right)_{f r}$ & $P_{f r}, \mathrm{kbar}$ & $\begin{array}{c}\sigma^{\prime}(T), \\
10^{-6} \mathrm{~J} /\left(\mathrm{m}^{2} \cdot \mathrm{K}\right)\end{array}$ & $\begin{array}{c}\sigma^{\prime}(P), \\
10^{-3} \mathrm{~J} /\left(\mathrm{m}^{2} \cdot \mathrm{kbar}\right)\end{array}$ \\
\hline \multirow{2}{*}{300} & 0.64553 & 4396.78 & 16683.0 & -4.488 & 0.34854 & 35589.9 & -0.054 & -0.708 \\
\hline & 0.64778 & 1918.97 & 7246.0 & -25.694 & 0.34966 & 15476.4 & -2.455 & -0.707 \\
\hline \multirow{2}{*}{3000} & 0.64721 & 4566.15 & 16393.3 & -137.177 & 0.35003 & 35428.3 & -185.3 & -0.705 \\
\hline & 0.65284 & 2103.60 & 6916.0 & -139.451 & 0.35377 & 15318.5 & -198.8 & -0.701 \\
\hline
\end{tabular}
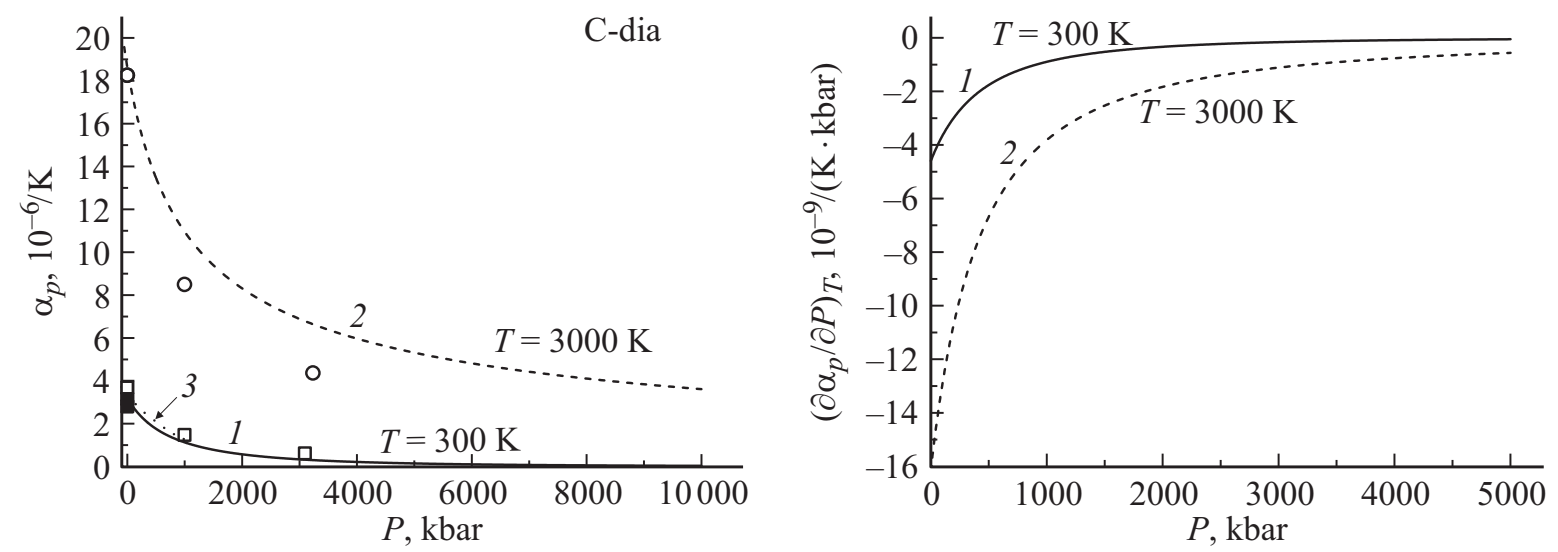

Рис. 3. Изотермы зависимости $\sigma_{p}(P)-$ коэффициента теплового расширения $\left(\right.$ в $\left.10^{-6} / \mathrm{K}\right)-$ слева и изотермы зависимости $\sigma_{p}^{\prime}(P)$ - производной $\alpha_{p}(P)$ по давлению (в $\left.10^{-9} /(\mathrm{K} \cdot \mathrm{kbar})\right)-$ справа.
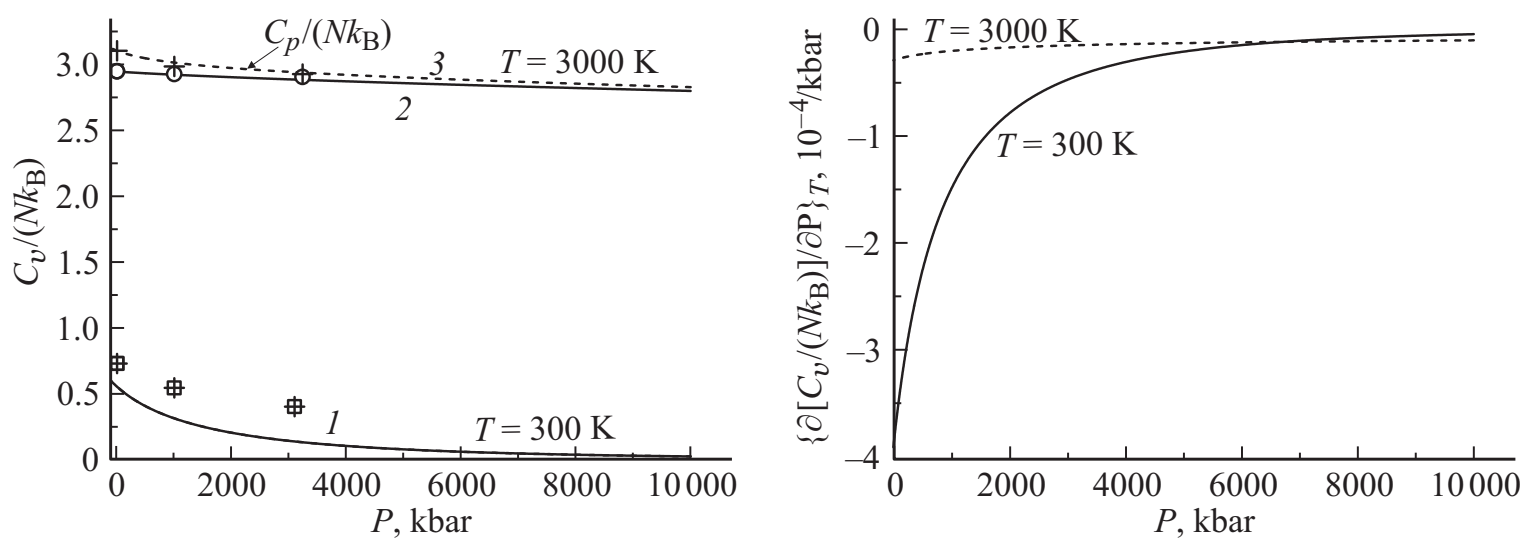

Рис. 4. Слева - изотермы барических зависимостей удельных теплоемкостей алмаза. Линиями показаны рассчитанные нами зависимости для $C_{v} /\left(N k_{B}\right)$ - изохорной теплоемкости (сплошные линии: 1 - для $300 \mathrm{~K}$ и $2-$ для $\left.3000 \mathrm{~K}\right)$ и для $C_{p} /\left(N k_{B}\right)-$ изобарной теплоемкости (штриховая линия 3 для $3000 \mathrm{~K}$ ). Справа - рассчитанные нами барические зависимости для производной изохорной теплоемкости по давлению (в $10^{-4} / \mathrm{kbar}$ ) вдоль изотерм: $300 \mathrm{~K}$ (нижняя сплошная линия) и $3000 \mathrm{~K}$ (верхняя штриховая линия). 
видно, что при $P \rightarrow \infty$ функция $\alpha_{p}(P)$ стремится к постоянной величине, а функция $\alpha_{p}^{\prime}(P)$ стремится к нулю.

На рис. 4 слева показаны изотермы барических зависимостей удельных теплоемкостей алмаза как для $C_{v} /\left(N k_{B}\right)$, так и для $C_{p} /\left(N k_{B}\right)$. Сплошными линиями показаны рассчитанные нами зависимости для $C_{v} /\left(N k_{B}\right)-$ изохорной теплоемкости: 1 - для $300 \mathrm{~K}$ и 2 для $3000 \mathrm{~K}$. Штриховая линия 3 изотерма $C_{p} /\left(N k_{B}\right)-$ изобарной теплоемкости для $3000 \mathrm{~K}$. При $300 \mathrm{~K}$ изотерма $C_{p}(P)$ сливается с $C_{v}(P)$. На левом графике также показаны результаты из работы [2, табл. 1А]: нижние символы - для $298.15 \mathrm{~K}$, верхние - для $3000 \mathrm{~K}$; квадраты и кружки - результаты для $C_{v} /\left(N k_{B}\right)$, крестики - для $C_{p} /\left(N k_{B}\right)$. На рис. 4 справа показаны изотермы для производной изохорной теплоемкости по давлению (в $\left.10^{-4} / \mathrm{kbar}\right)$. Из рис. 4 видно, что при $P \rightarrow \infty$ функции $C_{v}(P)$ и $C_{p}(P)$ стремятся к постоянной величине, а функция $C_{v}^{\prime}(P)$ и разность $C_{p}(P)-C_{v}(P)$ стремятся к нулю. Независимость $C_{v}$ от давления при $T>3000 \mathrm{~K}$ есть следствие классического закона Дюлонга-Пти: $C_{v}(T \gg \Theta)=3 N k_{B}$. Исчезноваение разности $C_{p}(P)-C_{v}(P)$ получается из-за уменьшения с давлением коэффициента теплового расширения (см. рис. 3) и первого параметра Грюнайзена (см. (11)).

Зависимость $\sigma(100)$ - удельной поверхностной энергии грани $(100)$ и $\sigma^{\prime}(T)=(\partial \sigma / \partial T)_{V}-$ ее изохорной производной по температуре от степени сжатия $\left(V / V_{0}\right)$ алмаза вдоль изотерм 300 и $3000 \mathrm{~K}$ при использовании глубины потенциала $D_{s}$ была представлена нами в [13]. Было показано, что функция $\sigma\left(V / V_{0}\right)$ при сжатии сначала возрастает до величины $\sigma(100)_{\max }$, а потом резко уменьшается, переходя при $\left(V / V_{0}\right)_{f r}$ в отрицательную область: $\sigma\left(V / V_{0}\right)_{f r}=0$. Легко понять, что при $\sigma<0$ структура кристалла будет распадаться на домены, увеличивая площадь междоменной поверхности.

В табл. 2 представлены рассчитанные значения $\sigma(100)$ и ее производных как по температуре: $\sigma^{\prime}(T)=$ $=(\partial \sigma / \partial T)_{V}$, так и по давлению: $\sigma^{\prime}(P)=(\partial \sigma / \partial P)_{T}$, в четырех характеристических точках изотермической зависимости $\sigma(P)$ : в точке $P=0$, в точке $V / V_{0}=1$, в точке максимума $\sigma(100)_{\max }$ и в точке фрагментации $\sigma(100)_{f r}=0$. Для каждой температуры в первой строке представлены результаты расчетов с глубиной потенциала $D_{b}$, т.е. для упругой (обратимой) деформации, а во второй строке - результаты расчетов с $D_{s}$, т.е. для пластической (необратимой) деформации алмаза.

Известные из литературы оценки величины $\sigma(100)$ для алмаза при нормальных условиях лежат в интервале [3,19]: $\sigma(100)=5710-9300\left[10^{-3} \mathrm{~J} / \mathrm{m}^{2}\right]$. Из сравнения с данными табл. 2 видно, что лучше согласуются с данными оценками результаты, полученные с величиной $D_{s}$, т. е. с потенциалом для пластической (необратимой) деформации кристалла. Расчеты показали, что при $P \rightarrow \infty$ функция $\sigma^{\prime}(P)$ экспоненциально убывает и стремится к постоянной отрицательной величине. Это указывает на то, что при $P \rightarrow \infty$ функция $\sigma(P)<0$ убывает по линейной зависимости.

\section{Заключение}

1. При использовании корректной функциональной зависимости для температуры Дебая $\Theta\left(V / V_{0}\right)$ и самосогласованно определенных четырех параметров межатомного потенциала Ми-Леннард-Джонса (1), как уравнение состояния, так и барическую зависимость термодинамических свойств алмаза можно рассчитать в рамках сравнительно простой аналитической модели без каких-либо подгоночных параметров.

2. Получены барические зависимости следующих свойств алмаза: $B_{T}-$ изотермического модуля сжатия и $B^{\prime}(P), C_{v}$ - изохорной теплоемкости и $C_{v}^{\prime}(P), C_{p}-$ изобарной теплоемкости, $\alpha_{p}$ - коэффициента теплового расширения и $\alpha_{p}^{\prime}(P), \sigma-$ удельной поверхностной энергии, а также производных $\sigma^{\prime}(P)$ и $\sigma^{\prime}(T)$. Расчеты, выполненные вдоль двух изотерм 300 и $3000 \mathrm{~K}$ вплоть до $P=10000 \mathrm{kbar}=1000 \mathrm{GPa}$, т. е. до $V / V_{0}=0.5$, показали хорошее согласие с экспериментальными данными.

3. Показано, что при $P \rightarrow \infty$ функции $B_{T}(P)$ и $\sigma(P)<0$ изменяются по линейной зависимости, функции $B^{\prime}(P), \alpha_{p}(P), C_{v}(P), C_{p}(P)$ и $\sigma^{\prime}(P)$ стремятся к постоянным величинам, а функции $\alpha_{p}^{\prime}(P), C_{v}^{\prime}(P)$ и разность $C_{p}(P)-C_{v}(P)$ стремятся к нулю.

4. Ранее в рамках данной модели удалось получить хорошее согласие с экспериментом также и для различных кристаллических модификаций железа: для ОЦК$\mathrm{Fe}[20]$ и для ГЦК-Fe [21]. Поэтому полученные здесь результаты для алмаза указывают на то, что это не случайное удачное совпадение результатов расчета с экспериментальными данными, а хорошая работоспособность разработанной методики расчета.

Автор выражает благодарность проф. П.И. Дорогокупцу, К.Н. Магомедову, 3.М. Сурхаевой и М.М. Гаджиевой за плодотворные дискуссии и помощь в работе.

Работа выполнена при финансовой поддержке РФФИ в рамках научного проекта № 16-03-00041_а и Программы Президиума РАН (программа № I.11П(1)).

\section{Список литературы}

[1] Holzapfel W.B. // High Pressure Res. 2010. Vol. 30. N 3. P. 372-394.

[2] Дорогокупеи, П.И., Соколова Т.С., Данилов Б.С., Литасов К.Д. // Геодинамика и Тектонофизика. 2012. Т. 3. Вып. 2. С. 129-166.

[3] Магомедов М.Н. Изучение межатомного взаимодействия, образования вакансий и самодиффузии в кристаллах. М.: Физматлит, 2010. 544 с.

[4] Магомедов М.Н. // ЖТФ. 2013. Т. 83. Вып. 9. С. 56-62.

[5] Жирибалько Л. Статистическая физика твердого тела: Пер. с англ. М.: Мир, 1975. 383 с. [Girifalco L.A. Statistical Physics of Materials. N. Y.: J. Wiley and Sons Ltd., 1973.]

[6] Магомедов М.Н. // ФТТ. 2004. Т. 46. Вып. 5. С. 924-937.

[7] Магомедов М.Н. // Журн. неорг. химии. 2004. Т. 49. Вып. 12. С. 2057-2067.

[8] Александров И.В., Гончаров А.Ф., Зисман А.Н., Стишов С.М. // ЖЭТФ. 1987. Т. 93. Вып. 8. С. 680-691. 
[9] Магомедов М.Н. // ТВТ. 2009. Т. 47. № 3. С. 379-387.

[10] Новикова С.И. Тепловое расширение твердых тел. М.: Наука. 1974. 294 с.

[11] Slack G.A., Bartram S.F. // J. Appl. Phys. 1975. Vol. 46. N 1. P. 89-98.

[12] Reeber R.R., Wang K. // J. Electron. Mater. 1996. Vol. 25. N 1. P. 63-67.

[13] Магомедов М.Н. // ЖТФ. 2013. Т. 83. Вып. 12. С. 87-96.

[14] Bradley D.K., Eggert J.H., Smith R.F., Prisbrey S.T., Hicks D.G., Braun D.G., Biener J., Hamza A.V., Rudd R.E., Collins G.W. // Phys. Rev. Lett. 2009. Vol. 102. N 7. P. 075503 (1-4).

[15] Vinet P., Ferrante J., Rose J.H., Smith J.R. // J. Geophys. Res. 1987. Vol. 92. N B9. P. 9319-9325.

[16] Occelli F., Loubeyre P., Letoullec R. // Nature Mater. 2003. Vol. 2. N 3. P. $151-154$.

[17] Бацанов С.С. Структурная химия. Факты и зависимости. М.: Диалог-МГУ, 2000. 292 с.

[18] Xie J., Chen S.P., de Gironcoli S., Baroni S. // Phys. Rev. B. 1999. Vol. 60. N 13. P. 9444 (1-4).

[19] Stekolnikov A.A., Bechstedt F. // Phys. Rev. B. 2005. Vol. 72. N 12. P. $125326(1-9)$.

[20] Магомедов М.Н. // ЖТФ. 2015. Т. 85. Вып. 11. С. 48-54.

[21] Магомедов М.Н. // ЖТФ. 2017. Т. 87. Вып. 4. С. 549-556. 